\title{
AJUSTE DE CURVAS Y CORRELACIÓN DE VARIABLES EN EL LABORATORIO DE FÍSICA
}

Moisés Segundo Sánchez Arteaga ${ }^{1}$

\section{RESUMEN}

En el presente trabajo se muestra que es posible obtener una correlación entre las variables que participan en los fenómenos físicos, partiendo del uso de las ecuaciones dimensionales, y tomando en cuenta datos experimentales se puede establecer que la linealización entre las variables, es la más adecuada, lo cual permite comprobar las relaciones de las variables en un experimento obtenidas por las leyes físicas y además calcular parámetros participantes en el fenómeno.

Palabras claves: Linealización de variables, correlación de datos, ajuste de curvas, experimentos de física.

\section{SUMMARY}

In the present work it shows that it is possible to obtain a correlation between the variables involved in physical phenomena, based on the use of dimensional equations, and taking into account experimental data can be established that the linearization between variables, is the most appropriate, which lets you check the relationships of the variables in an experiment obtained by physical laws and calculate besides parameters involved in the phenomenon.

Keywords: Physical model, simulation, physical laws, equation solving.

\section{INTRODUCCIÓN}

En los experimentos realizados en las asignaturas de física así como también en las ciencias en general cuando se trata de encontrar una relación entre variables participantes en un fenómeno natural, el métodos científico nos conduce primeramente a analizar y encontrar las variables físicas que participan en los experimentos así como también obtener una expre-

Área Académica de Física. Facultad de Ingeniería. Universidad Ricardo Palma. moises.sancheza@urp.pe 
sión algebraica de la interdependencia de las variables. Esto se puede lograr inicialmente haciendo uso de las ecuaciones dimensionales.

Posteriormente se debe contar con información cuantitativa del experimento en la que se midan las variables participantes y se haga una tabla de datos con las unidades correspondientes, en la que se recomienda el uso del sistema internacional de unidades (S.I.).

Con la expresión resultante de las ecuaciones dimensionales y con los datos obtenidos en la realización del experimento, se debe llevar a cabo las gráficas correspondientes y tratar de linealizarlas afín de poder tener certeza de la correlación matemática entre las variables y utilizar la ecuación matemática de la recta para el cálculo de algunos constantes importantes características del fenómeno.

Por lo general las gráficas resultantes son curvas las cuales pueden tener diferentes formas de expresar la ecuación de correlación correspondiente, como por ejemplo expresión polinómica de diferentes grados (cuadrática, cubica, etc.), exponencial, logarítmica, potencias entre otras. Desde el punto de vista estadístico, aquella que tuviese mayor coeficiente de determinación $\left(\mathrm{R}^{2}\right)$, corresponderá a un mejor ajuste de curvas; sin embargo desde el punto de vista del análisis cualitativo del fenómeno, estas graficas no nos permite analizar y comprender la presencia de las diferentes variables físicas presentes en el fenómeno y que estén de acuerdo con las ecuaciones provenientes de las leyes físicas.

Es por ello que se nos hace necesario identificar las gráficas de las variables con las leyes de la física según nos resulte del análisis dimensional y buscar una relación lineal lo cual nos permitirá verificar la certeza de la formulación y por otro lado a través de la ecuación de la relación lineal con el uso del intercepto con el eje y de la pendiente de la ecuación lineal numérica podremos obtener numéricamente los valores de los diferentes parámetros participantes en el fenómeno.

En la presente publicación se presentan varios casos de experimentos de física en la cual con el uso inicial de ecuaciones dimensionales se establecerá una correlación entre las variables del experimento y que luego de la linealización con los datos experimentales podremos corroborar la relación entre las cantidades físicas y cuantificar los diferentes parámetros participantes. 


\section{MATERIAL Y MÉTODOS}

Se presentan varios casos típicos en el análisis de datos de experimentos realizados en la asignatura de física general.

\section{Movimiento rectilíneo uniformemente variado (MRUV)}

Al soltar cierto cuerpo desde el reposo por un plano inclinado, este desciende en forma acelerada por el plano, cambiando la posición en función de la aceleración y del tiempo. Es de interés conocer la relación entre sus cantidades posición, aceleración y tiempo.

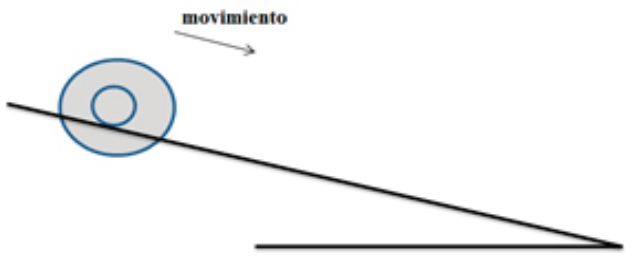

\section{Uso de ecuaciones dimensionales}

Para obtener una relación entre las variables posición (x) en función de la aceleración (a) y el tiempo (t), planteamos la siguiente ecuación:

$\mathrm{x}=(\mathrm{a})^{\mathrm{p}}(\mathrm{t})^{\mathrm{q}}$

siendo $[\mathrm{x}]=\mathrm{L} ;[\mathrm{a}]=\mathrm{LT}^{-2} \mathrm{y}[\mathrm{t}]=\mathrm{T}$; reemplazando en la ec. (1), resulta la siguiente ecuación dimensional:

$$
[L]=\left[L T^{-2}\right]^{p}[T]^{q} ; \quad[L]^{1}=[L]^{p} T^{q-2}
$$

Obteniéndose de esta ecuación:

$\mathrm{p}=1$ y q-2 $=0$ ó $\mathrm{q}=2$; reemplazando en la ec. (1) resulta la siguiente ecuación de correlación:

$\mathrm{x}=\mathrm{Ct}^{2}$

siendo $\mathrm{C}$ una constante adimensional

\section{Uso de datos experimentales}

Mostramos los datos obtenidos en el experimento con un máximo de tres cifras significativas y por consiguiente para un análisis de estas variables, 
graficamos la variable posición $\mathrm{x}$ en función de $\mathrm{t} y$ también de $\mathrm{t}^{2}$ afín de realizar una comparación con la ec.(2).

\begin{tabular}{|c|c|c|}
\hline$x(\mathrm{~cm})$ & $\mathbf{t}(\mathbf{s})$ & $\mathbf{t}^{\mathbf{2}}\left(\mathbf{s}^{\mathbf{2}}\right)$ \\
\hline 0 & 0,0 & 0,0 \\
\hline 7,5 & 3,9 & 15 \\
\hline 15,0 & 5,8 & 34 \\
\hline 22,5 & 7,3 & 53 \\
\hline 30,0 & 8,5 & 72 \\
\hline 37,5 & 9,4 & 89 \\
\hline 45,0 & 10,4 & 107 \\
\hline 52,5 & 11,3 & 127 \\
\hline 60,0 & 12,1 & 147 \\
\hline
\end{tabular}
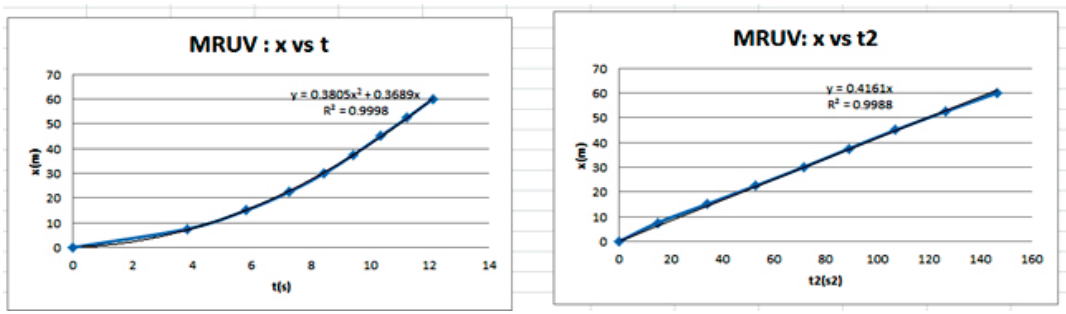

De acuerdo con la ecuación (2), la gráfica $\mathrm{x} v \mathrm{v} \mathrm{t}^{2}$, representa físicamente al movimiento en estudio.

De las ecuaciones de la cinemática y de la ecuación (2) para la posición (x), se tiene:

Velocidad: $v=\frac{d x}{d t}=2 C t$

Aceleración: $a=\frac{d v}{d t}=2 C$

De la ecuación de la gráfica $\mathrm{x}$ vs $\mathrm{t}^{2} \mathrm{y}$ la ec. (2). Se obtiene: $\mathrm{C}=$ pendiente $=0,4161\left(\mathrm{~m} / \mathrm{s}^{2}\right)$;

Resultando la aceleración de móvil: $\mathrm{a}=2 \mathrm{C}=0,832 \mathrm{~m} / \mathrm{s}^{2}$

Se trata de un Movimiento uniformemente variado con aceleración constante, $\mathrm{a}=0,832 \mathrm{~m} / \mathrm{s}^{2}$ y siendo la ecuación de la correlación de variables del experimento:

$$
x=\frac{1}{2} a t^{2}=0,416 t^{2}
$$




\section{Movimiento de un proyectil}

Al lanzarse horizontalmente un proyectil, su trayectoria y las posiciones $(\mathrm{x}, \mathrm{y})$ van a depender de la altura inicial $(\mathrm{H})$, velocidad inicial $\left(\mathrm{v}_{0}\right)$, aceleración de la gravedad y del tiempo. Es de interés conocer la relación entre estas cantidades.

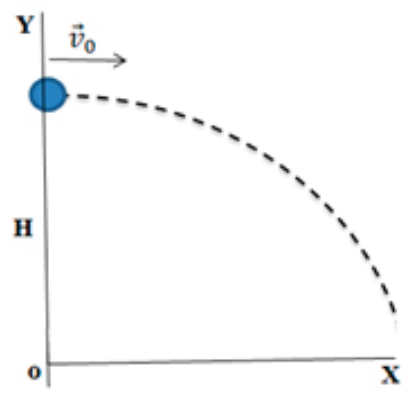

\section{Uso de ecuaciones dimensionales}

Para obtener una relación entre las variables altura $(\mathrm{y})$, posición $(\mathrm{x})$, aceleración de la gravedad (a) y velocidad inicial horizontal $\mathrm{v}_{0}$, se plantea la siguiente ecuación:

$\mathrm{y}-\mathrm{y}_{0}=\left(\mathrm{v}_{0}\right)^{\mathrm{p}}(\mathrm{a})^{\mathrm{q}}(\mathrm{x})^{\mathrm{r}}$

siendo $[\mathrm{x}]=[\mathrm{y}]=\mathrm{L} ;[\mathrm{v}]=\mathrm{LT}^{-1} \mathrm{y}[\mathrm{a}]=\mathrm{LT}^{-2}$, reemplazando en la ec.(4), obtenemos la siguiente ecuación dimensional

$$
[L]=\left[L T^{-1}\right]^{p}\left[L T^{-2}\right]^{q}[L]^{r}
$$

resultando de esta ecuación: $1=\mathrm{p}+\mathrm{q}+\mathrm{r} ; 0=-\mathrm{p}-2 \mathrm{q}$; resolviendo y considerando las ecuaciones cinemáticas, se obtiene $: \mathrm{p}=-2, \mathrm{q}=1, \mathrm{r}=2$; reemplazando en la ec. (4) resulta la siguiente ecuación de correlación:

$$
y=H-\frac{g}{v_{0}^{2}} x^{2}
$$

\section{Uso de datos experimentales}

Mostramos los datos obtenidos en el experimento con un máximo de tres cifras significativas y por consiguiente para un análisis de estas variables, 
graficamos la variable altura $(\mathrm{y})$ versus posición $(\mathrm{x}) \mathrm{y}$ también versus $\mathrm{x}^{2}$ afín de realizar una comparación con la ec. (5).

\begin{tabular}{|c|c|c|}
\hline $\mathbf{x}(\mathbf{m})$ & $\mathbf{y}(\mathbf{m})$ & $\mathbf{x}^{\mathbf{2}}\left(\mathbf{m}^{\mathbf{2}} \mathbf{)}\right.$ \\
\hline 0,0 & 8,0 & 0,0 \\
\hline 1,2 & 7,8 & 1,4 \\
\hline 2,6 & 7,2 & 6,8 \\
\hline 3,9 & 6,2 & 15 \\
\hline 5,2 & 4,8 & 27 \\
\hline 6,5 & 3,1 & 42 \\
\hline 7,8 & 0,9 & 60 \\
\hline
\end{tabular}
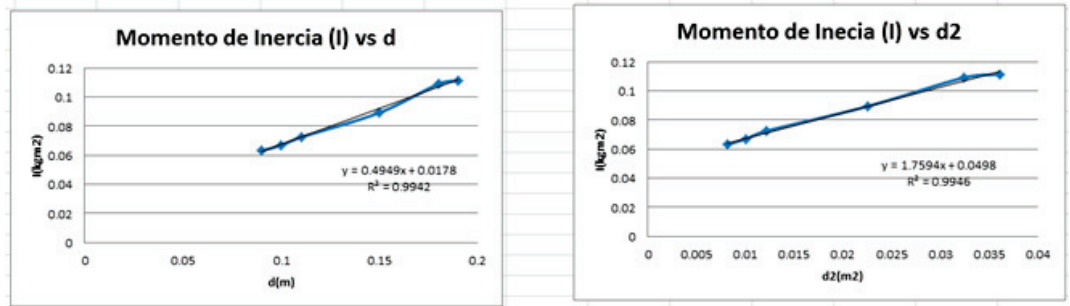

Podemos apreciar de las gráficas que la correspondiente a nuestro experimento es la recta, la cual es concordante con la ecuación (5): $y=H-\frac{g}{2 v_{0}^{2}} x$ Intercepto $=$ altura inicial $(H)=7,98 \mathrm{~m}$

pendiente $=-\frac{g}{2 v_{o}^{2}}=-0,1163$

Obteniéndose la velocidad inicial de lanzamiento: $\mathrm{v}_{0}=6,49 \mathrm{~m} / \mathrm{s}$

\section{Péndulo Físico}

Un cuerpo rígido que oscila alrededor de algún punto es un péndulo físico. El caso en estudio es el de una barra rígida que oscila alrededor de un punto "O" a una distancia "d" de su centro de masa (G).

El momento de inercia de la barra depende de la masa M, y la distancia del centro de masa con respecto al eje de giro

\section{Uso de ecuaciones dimensionales}

Para obtener una relación entre el momento

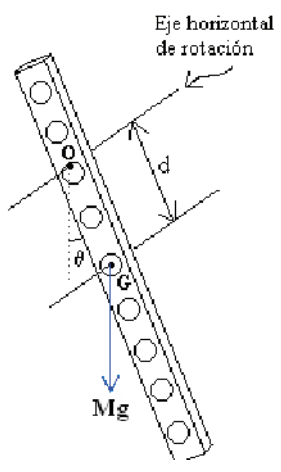
de inercia (I), Masa de la barra (M), distancia entre el punto de giro y el 
centro de masa (d), se plantea la siguiente ecuación:

$\mathrm{I}=(\mathrm{M})^{\mathrm{p}}(\mathrm{d})^{\mathrm{q}}$

Siendo: $[\mathrm{I}]=\left[\mathrm{ML}^{2}\right],[\mathrm{d}]=\mathrm{L}$

Reemplazando en ec.(7), se obtiene la siguiente ecuación dimensional

$$
\left[M L^{2}\right]=[M]^{p}[L]^{q}
$$

resultando: 1 = p; 2 = q; resolviendo y reemplazando en la ec.(7), obtenemos la ecuación de correlación de las variables del experimento:

$$
I=M d^{2}
$$

\section{Uso de datos experimentales}

Mostramos los datos obtenidos en el experimento con un máximo de tres cifras significativas y por consiguiente para un análisis de estas variables, graficamos las variables momento de inercia (I) versus distancia (d) y también versus $\mathrm{d}^{2}$ afín hacer una comparación con la ec. (8). El momento de Inercia para cada posición "d". lo calculamos a partir del Periodo y la masa, con la siguiente ecuación. $T=2 \pi \sqrt{I / M g d}$

\begin{tabular}{|c|c|c|c|}
\hline $\mathbf{d}(\mathbf{m})$ & $\mathbf{T}(\mathbf{s})$ & $\mathbf{I}\left(\mathrm{kgm}^{2}\right)$ & $\mathbf{d}^{2}\left(\mathbf{m}^{2}\right)$ \\
\hline 0,09 & 1,27 & 0,063 & 0,008 \\
\hline 0,10 & 1,24 & 0,067 & 0,010 \\
\hline 0,11 & 1,23 & 0,072 & 0,012 \\
\hline 0,15 & 1,17 & 0,089 & 0,023 \\
\hline 0,18 & 1,18 & 0,109 & 0,032 \\
\hline 0,19 & 1,16 & 0,111 & 0,036 \\
\hline
\end{tabular}
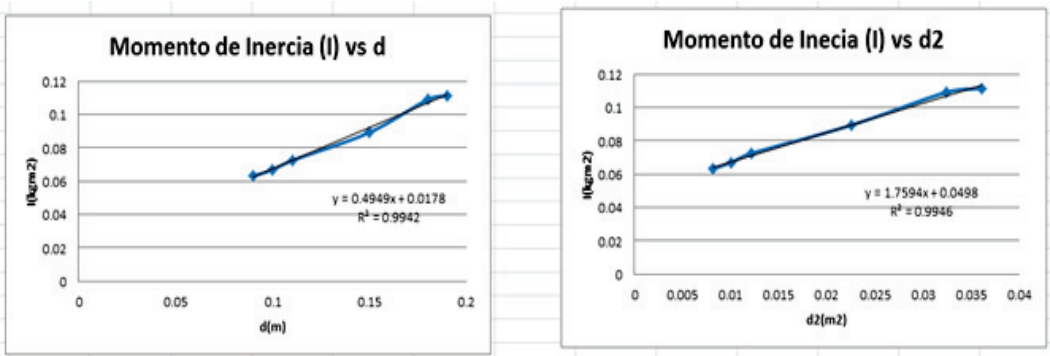
Apreciando las gráficas podemos concluir que la gráfica que más se ajusta a la ecuación (8) es la gráfica I vs d $\mathrm{d}^{2}$; lo cual corrobora la ecuación obtenida por las leyes físicas, conocida como Teorema de Steiner o Teorema de ejes paralelos: $\mathrm{I}=\mathrm{I}_{\mathrm{CM}}+\mathrm{Md}^{2}$,

Por comparación entre la ecuación física y la ecuación de ajuste de curvas, se obtiene:

$$
\text { Intercepto }=\text { Momentode Inercia respecto al CM }\left(I_{C M}\right)=0,0498 \mathrm{kgm}^{2}
$$

pendiente $=$ Masa de la barra $(M)=1,76 \mathrm{~kg}$

\section{Oscilación sistema masa - resorte}

En la oscilación de un sistema masa - resorte, el periodo y la frecuencia de oscilación dependen de la constante elástica del resorte $(\mathrm{K})$ y de la masa $(\mathrm{M})$.

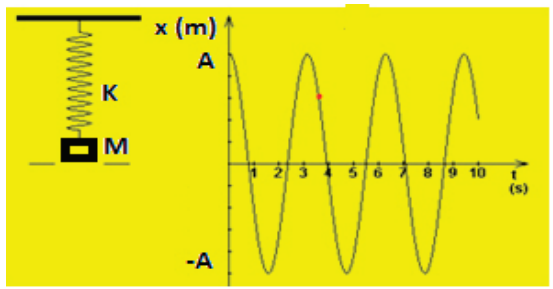

\section{Uso de ecuaciones dimensionales}

Para obtener una relación entre la frecuencia angular (W), la masa del bloque $(\mathrm{M})$, y la constante elástica del resorte $(\mathrm{K})$, se plantea la siguiente ecuación:

$\mathrm{W}=(\mathrm{K})^{\mathrm{p}}(\mathrm{M})^{\mathrm{q}}$

Siendo: $[\mathrm{W}]=\mathrm{T}^{-1} \mathrm{y}[\mathrm{K}]=\mathrm{MT}^{-2}$;

Reemplazando en la ec. (10), se obtiene la siguiente ecuación dimensional:

$$
\left[T^{-1}\right]=\left[M T^{-2}\right]^{p} M^{q}
$$


resultando: $-1=-2 \mathrm{p} ; 0=\mathrm{p}+\mathrm{q}$; resolviendo y considerando las ecuaciones cinemáticas, se obtiene: $\mathrm{p}=1 / 2 ; \mathrm{q}=-1 / 2$ y reemplazando en la ec.(10), se tiene la ecuacion de correlacion de las variables del experimento:

$$
W=\sqrt{\frac{K}{M}}
$$

\section{Uso de datos experimentales}

Se muestran los datos obtenidos en el experimento con un máximo de tres cifras significativas y por consiguiente para un análisis de estas variables, graficamos la variable frecuencia angular (W) versus $1 / \mathrm{M}$ y W vs. $1 / \sqrt{M}$, sin cambiar el resorte, afin de hacer una comparacion con la ec. (11)

\begin{tabular}{|c|c|c|c|c|}
\hline $\mathbf{M}(\mathbf{k g})$ & $\mathbf{T}(\mathbf{s})$ & $\mathbf{W}(\mathbf{r a d} / \mathbf{s})$ & $\mathbf{1} / \mathbf{M}^{\left(\mathbf{k g}^{\mathbf{- 1}}\right)}$ & $\sqrt{\mathbf{1} / \mathbf{M}^{(}}\left(\mathbf{k g}^{-\mathbf{1} / \mathbf{2})}\right.$ \\
\hline 0,10 & 0,386 & 16,3 & 10,0 & 3,16 \\
\hline 0,15 & 0,486 & 12,9 & 6,67 & $\mathbf{2 , 5 8}$ \\
\hline 0,20 & 0,521 & 12,1 & 5,00 & 0,20 \\
\hline 0,25 & 0,627 & 10,0 & 4,00 & 0,25 \\
\hline 0,30 & 0,666 & 9,4 & 3,33 & 1,83 \\
\hline
\end{tabular}
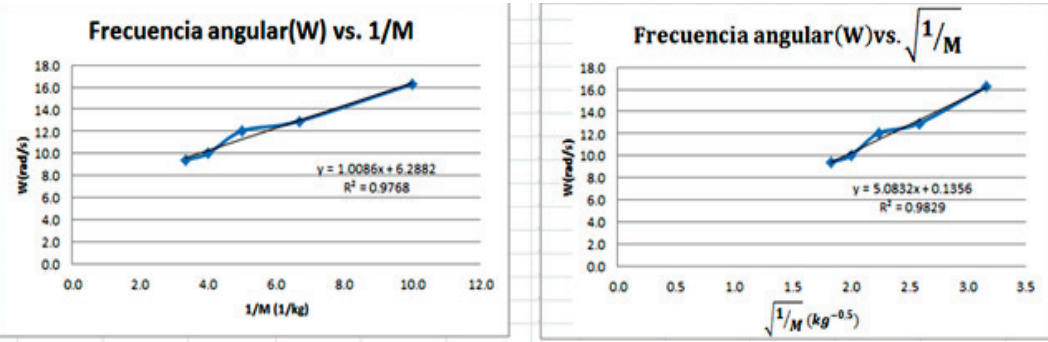

Observando las gráficas podemos concluir que la gráfica que más se ajusta a la ec. (11) según nuestros datos experimentales, por tener mayor coeficiente de determinación $\left(\mathrm{R}^{2}\right)$ es la gráfica $\mathrm{W}$ vs $1 / \sqrt{M}$; lo cual corrobora la ecuación obtenida por las leyes físicas.

Por comparación entre la ecuación física (ec. 11) y la ecuación de la recta, se obtiene:

Intercepto $=0,1356$ se toma como despreciable 
pendiente $=\sqrt{K}=5,0832 ;$ resultando: $\mathrm{K}=25,8 \mathrm{~N} / \mathrm{m}$

\section{Péndulo Simple}

En la oscilación de un péndulo simple, el periodo $(\mathrm{T})$ y la frecuencia de oscilación dependen de la longitud del resorte (L) y de la aceleración de la gravedad $(\mathrm{g})$

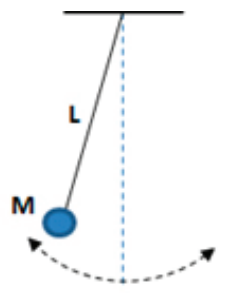

\section{Uso de ecuaciones dimensionales}

Para obtener una relación del periodo $(\mathrm{T})$ con las variables longitud (L), y la aceleración de la gravedad $(\mathrm{g})$, se plantea la siguiente ecuación:

$\mathrm{T}=(\mathrm{L})^{\mathrm{p}}(\mathrm{g})^{\mathrm{q}}$

Reemplazando las dimensiones, $[\mathrm{T}]=\mathrm{T},[\mathrm{L}]=\mathrm{L} \mathrm{y}[\mathrm{g}]=\mathrm{LT}^{-2}$, obtenemos la siguiente ecuación dimensional:

$$
[T]=[L]^{p}\left[L T^{-2}\right]^{q}
$$

resultando: $1=-2 \mathrm{q} ; 0=\mathrm{p}+\mathrm{q}$; resolviendo y considerando las ecuacionecinemáticas, se obtiene $: \mathrm{p}=1 / 2 ; \mathrm{q}=-1 / 2$.

Reemplazando en la ec. (13), se tiene la ecuacion de correlacion de las vartiables del experimento:

$$
T=C \sqrt{\frac{L}{g}}
$$

A continuación se muestran los datos obtenidos en el experimento con un máximo de tres cifras significativas y por consiguiente para un análisis de estas variables, graficamos la variable Periodo $(\mathrm{T})$ versus longitud del péndulo (L) y versus $\sqrt{L}$, afin de hacer una comparacion con la ec. (14) 


\begin{tabular}{|c|c|c|}
\hline $\mathbf{L}(\mathbf{m})$ & $\mathrm{T}(\mathbf{s})$ & $\sqrt{\mathrm{L}}\left(\mathbf{m}^{0.5}\right)$ \\
\hline & & \\
\hline 0.510 & 1.446 & 0.714 \\
\hline 0.575 & 1.536 & 0.758 \\
\hline 0.650 & 1.628 & 0.806 \\
\hline 0.710 & 1.700 & 0.843 \\
\hline 0.775 & 1.838 & 0.880 \\
\hline 0.900 & 1.912 & 0.949 \\
\hline 0.970 & 2.003 & 0.985 \\
\hline 1.140 & 2.148 & 1.068 \\
\hline
\end{tabular}
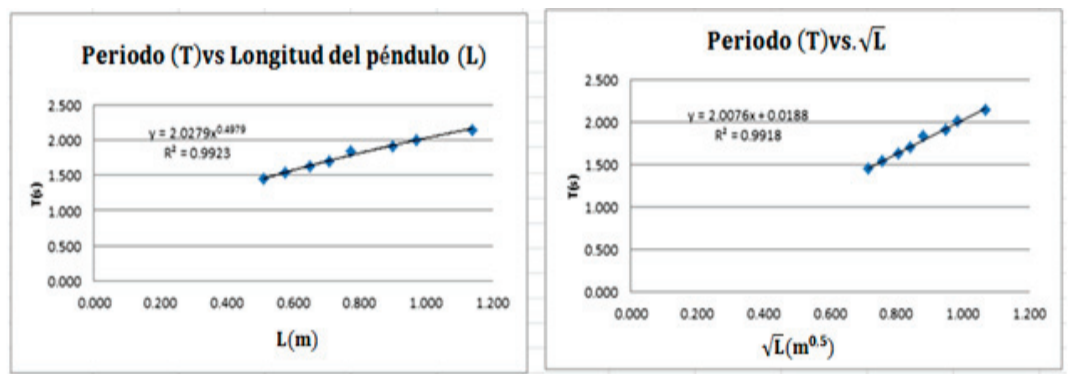

Apreciando las gráficas podemos concluir que ambas graficas confirman la ecuación (14). Utilizando la pendiente de la gráfica lineal, podemos calcular la constante $\mathrm{C}$, resultando:

$$
\frac{c}{\sqrt{g}}=2,0076 ; \mathrm{C}=6,28=2 \pi ; \mathrm{C}=6,28=2 \pi
$$

Reemplazando en la ec. (14). Se obtiene la ecuación para el péndulo simple:

$$
T=2 \pi \sqrt{\frac{L}{g}}
$$

\section{Fuerza de Flotación. Principio de Arquímedes}

En el experimento para encontrar una expresión de la fuerza de flotación (Empuje) con la densidad del líquido de inmersión y el volumen desplazado, se toman datos de la masa del cuerpo en el aire, del cuerpo inmerso en el fluido y del volumen desplazado. 

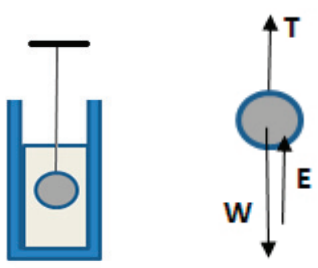

\section{Uso de ecuaciones dimensionales}

Para obtener una relación de la fuerza de empuje (E) con el volumen desplazado (V), densidad ( $\rho$ ) y la aceleración de la gravedad (g), se plantea la siguiente ecuación:

$\mathrm{E}=(\rho)^{\mathrm{p}}(\mathrm{V})^{\mathrm{q}}(\mathrm{g})^{\mathrm{r}}$

Reemplazando las dimensiones $[\mathrm{E}]=\mathrm{MLT}^{-2},[\rho]=\mathrm{ML}^{-3},[\mathrm{~V}]=\mathrm{L}^{3},[\mathrm{~g}]=$ $\mathrm{LT}^{-2}$ en la ec. (16), se obtiene la ecuación dimensional:

$$
\left[M L T^{-2}\right]=\left[M L^{-3}\right]^{p}\left[L^{3}\right]^{q}\left[L T^{-2}\right]^{r}
$$

resultando: $1=p ; 1=-3 p+3 q+r ;-2=-2 r$; resolviendo, resulta: $p=1 ; q=1$; $r=1$, reemplazando en la ec.(16), se obtiene la ecuacion de correlacion de las variables del experimento:

$$
E=\rho V g
$$

Se muestran los datos obtenidos en el experimento con un máximo de tres cifras significativas y por consiguiente para un análisis de estas variables, graficamos la variable Empuje (E) versus volumen desplazado (V).

\begin{tabular}{|c|c|c|c|}
\hline $\mathbf{M}(\mathbf{k g})$ & $\mathbf{V}(\mathbf{m} 3) \mathbf{x 1 0}(-3)$ & $\mathbf{W}(\mathbf{N})$ & $\mathbf{E}(\mathbf{N})$ \\
\hline 0.21 & 0.016 & 2.1 & 0.10 \\
\hline 0.5 & 0.059 & 4.9 & 0.49 \\
\hline 0.7 & 0.070 & 6.9 & 0.98 \\
\hline 1 & 0.097 & 9.8 & 1.08 \\
\hline 1.5 & 0.151 & 14.7 & 1.86 \\
\hline
\end{tabular}



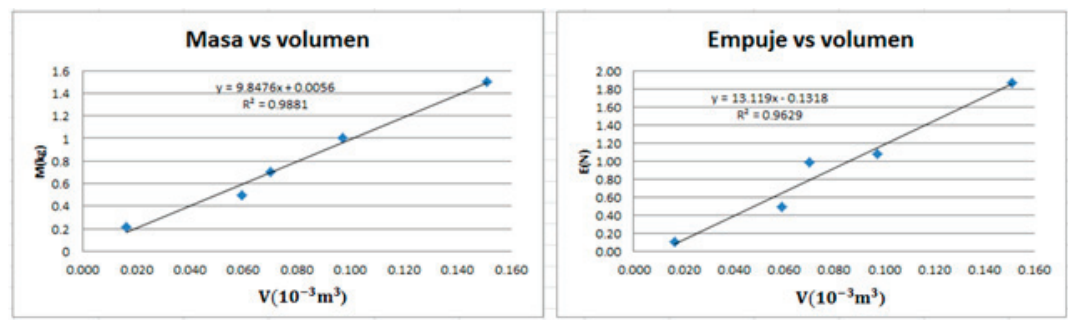

La primera grafica nos muestra la proporcionalidad entre la masa y el volumen del material del cuerpo, lo que nos permite conocer la densidad del material.

$$
M=\rho_{m} V ; \text { densidad }\left(\rho_{\mathrm{m}}\right)=\text { pendiente }=9,85 \times 10^{3} \mathrm{~kg} / \mathrm{m}^{3}
$$

La segunda gráfica, nos confirma la relación de la ec. (17) y nos permite hallar la densidad del líquido. De la ec. (17):

pendiente $=\rho g=13,1 \times 10^{3} \mathrm{~N} / \mathrm{m}^{3} ; \rho=1,35 \times 10^{3} \mathrm{~kg} / \mathrm{m}^{3}$

\section{RESULTADOS}

Podemos apreciar que es posible obtener una expresión matemática que correlacione las diferentes variables que participan en un fenómeno físico recurriendo en una primera parte a un análisis dimensional, lo que nos conlleva a un relación preliminar entre las variables y posteriormente, con datos obtenidos de la experimentación, gráficas y sus ecuaciones correspondientes podremos confirmar el resultado de las ecuaciones dimensionales así como también calcular algunos parámetros característicos del fenómeno.

\section{CONCLUSIONES}

De los ensayos experimentales mostrados y de las conclusiones obtenidas, podemos concluir que combinando un procedimiento analitico y otro experimental consistente en una formulación de las leyes participantes y una cuantificación de los datos tomados en la experimentación, se pueden hacer un correcto análisis en la búsqueda de las leyes y variables que participan en los fenómenos físicos en general y presentado la ecuación gobernante del fenómeno estudiado. 


\section{LITERATURA CITADA}

SEARS Y ZEMANSKY. 2013. 13 Edición. Física Universitaria. Volumen 1.

SEARS Y ZEMANSKY. 2013. Física Universitaria. Volumen 2.

Guías de Laboratorio de Física General - Facultad de Ingeniería - Universidad Ricardo Palma. Agosto del 2015. 